\section{Difference Between Familial and Sporadic Medullary Thyroid Carcinomas}

\section{To the Editor:}

A recent paper has focused the attention on the distinction of familial from sporadic medullary thyroid carcinomas (MTCs). ${ }^{3}$ This distinction is normally based on subtle morphologic changes, and it has been proven difficult for this and other pathologic conditions, such as melanocytic nevi associated with the atypical mole syndrome. ${ }^{3,6}$ The authors clearly conclude that there is no single morphologic criterion reliable for that distinction, although some findings tend to be more frequent in familial tumors. However, both diagnostic and biologic aspects would need additional comments for both C-cell hyperplasias $(\mathrm{CCH})$ and MTCs.

The presence of $\mathrm{CCH}$ is more frequently associated with, but not exclusive of, familial MTC. ${ }^{2,3}$ Although the authors present a classic morphologic classification of $\mathrm{CCH},{ }^{5}$ they do not draw any conclusion from that classification. The absence of $\mathrm{CCH}$ was found exclusive of sporadic MTC; the presence, however, was less informative. This positive finding is more discriminant for the sporadic/familial distinction when they are grouped into expansile (nodular and neoplastic) and nonexpansile (focal and diffuse; we will comment below on terminology). This grouping excludes almost half of $\mathrm{CCH}$-associated sporadic MTC. There is also a biologic support for that grouping. We studied MEN 2A-related $\mathrm{CCH}$ that were found to be consistently monoclonal with frequent microsatellite abnormalities in tumor suppressor genes, ${ }^{1}$ suggesting a C-cell intraepithelial neoplasia (C-CIN). These cases revealed either nodular or neoplastic growth patterns according with Perry et al. ${ }^{5}$ but did not reveal a different genetic pattern. These cases also showed significantly reduced apoptosis (as revealed by in situ end labeling) contributing to the cellular outgrowth. These two growth patterns share genetic (monoclonal with microsatellite abnormalities) and kinetic (downregulated apoptosis) profiles, which would justify grouping them as expansile patterns. No information is available on the genetic and kinetic features of focal or diffuse growth patterns and sporadic $\mathrm{CCH}$. The morphologic features and the data presented by Kaserer et al. ${ }^{2,3}$ also support grouping them as nonexpansile, at least until additional information proves otherwise.

Terminologically, two aspects need some clarification. First, focal and diffuse growth patterns are not fortunate descriptors. C-cell hyperplasia is always a focal process in the thyroid because it affects a certain number of follicles only. What can be focal is the follicular involvement. Probably focal and diffuse growth patterns would be better called segmental and circumferential when describing the follicular distribution. The second aspect is the term intraepithelial neoplasia. The expansile growth patterns of $\mathrm{CCH}$ share reproducible morphologic features, genetic and kinetic profiles consistent with C-CIN, but we do not have such an evidence for nonexpansile $\mathrm{CCH}$ yet. We would therefore propose to restrict the term $\mathrm{C}-\mathrm{CIN}$ to expansile $\mathrm{CCH}$ only (nodular and neoplastic growth patterns).

All these data support the neoplastic transformation of $\mathrm{C}$ cells through multiple steps and the morphologic sequence of intraepithelial-invasive neoplasm. Several reports provide information on the utility of basement membrane immunohistochemistry to distinguish intraepithelial from invasive neoplasms. ${ }^{3,4}$ The identification of early stromal invasion is more frequent after implementing pentagastrin and genetic screening for $\mathrm{C}$-cell lesions in families carrying RET germline mutations. However, the identification of this early stromal invasion documented by defects in the collagen IV layer accompanied by focal reduplication of basement membrane does not mean that the neoplasm has already acquired a metastatic potential. Kaserer et al. ${ }^{3}$ found lymph node metastases in tumors with desmoplastic stroma only. This finding highlights that a subclassification of these MTCs is still required. A similar example is documented for skin malignant melanomas with the distinction between radial and vertical growth phases, the former having absent or minimal potential for metastatic disease. In that sense, the desmoplastic stromal reaction to the MTC can help identify neoplasms with metastatic potential (so-called vertical growth phase).

In conclusion, the growth pattern of intraepithelial and invasive $\mathrm{C}$-cell proliferation can provide clues on the familial background. Multifocal and bilateral invasive tumors associated with expansile intraepithelial C-cell neoplasm would be familial CCH MTC in $98.13 \%$ of cases, according with data presented by Kaserer et al. ${ }^{3}$ The probability of being sporadic that neoplasm would be $0.1875 \times$ (multifocal) $\times 0.2143$ (bilateral $) \times 0.4643($ expansile $\mathrm{CCH})=0.0187$, or $1.87 \%$. Invasive MTCs still require the identification of tumors with metastatic potential; our personal experience and the Kaserer et al. data ${ }^{3}$ suggest that neoplasms with 
desmoplastic stromal reaction have more likely acquired that potential and would be in so-called vertical growth phase.

Lisa Mears, M.B.B.S.

Department of Histopathology and Morbid Anatomy, Barts London NHS Trust

London, U.K.

Salvador J. Diaz-Cano, M.D., Ph.D., M.R.C.Path. Department of Histopathology and Morbid Anatomy, Barts London NHS Trust

London School of Medicine and Dentistry

London, U.K.

\section{REFERENCES}

1. Diaz-Cano SJ, de Miguel M, Blanes A, et al. Germline RET 634 mutation positive MEN 2A-related C-cell hyperplasias have genetic features consistent with intraepithelial neoplasia. J Clin Endocrinol Metab 2001;86:3948-57.

2. Kaserer K, Scheuba C, Neuhold N, et al. C-cell hyperplasia and medullary thyroid carcinoma in patients routinely screened for serum calcitonin. Am J Surg Pathol 1998;22:722-8.

3. Kaserer K, Scheuba C, Neuhold N, et al. Sporadic versus familial medullary thyroid microcarcinoma: a histopathologic study of 50 consecutive patients. Am J Surg Pathol 2001;25:1245-51.

4. McDermott MB, Swanson PE, Wick MR. Immunostains for collagen type IV discriminate between C-cell hyperplasia and microscopic medullary carcinoma in multiple endocrine neoplasia, type 2a. Hum Pathol 1995;26:1308-12.

5. Perry A, Molberg K, Albores-Saavedra J. Physiologic versus neoplastic C-cell hyperplasia of the thyroid: separation of distinct histologic and biologic entities. Cancer 1996;77:750-6.

6. Pozo L, Naase M, Cerio R, et al. Critical analysis of histologic criteria for grading atypical (dysplastic) melanocytic nevi. Am J Clin Pathol 2001;115:194-204.

\section{Board Certification in Anatomic Pathology}

\section{To the Editor:}

The 2002 meeting of the Association of Directors of Anatomic and Surgical Pathology (ADASP), held in February in Chicago, focused on "Board Certification in Surgical Pathology," a topic that potentially has profound implications for the entire anatomic pathology community. This issue had been informally and incompletely discussed at prior ADASP meetings. The purpose now was to attempt to discuss this issue in depth. Many reasons for the need for a subspecialty board in surgical pathology have been given in the past. Many have noted that the cytopathology board certification "with added qualification" seemed to bring recognition and prestige to that subspecialty. Why then could not the same thing

The authors are currently ADASP officers: Dr. Smith, Secretary/Treasurer; Dr. Connolly, President; Dr. Fletcher, President-elect. This report has been approved by the ADASP Council.

This report will be published in both Human Pathology and the American Journal of Surgical Pathology. be done for surgical pathology? Some noted that the surgical pathologist lacked specific recognition; therefore, board certification would help serve as verification and recognition of the de facto subspecialty. Others maintain that certification would provide recognition of how most pathologists spend the majority of their professional time. There are many books and postgraduate courses with the words "surgical pathology" in their titles. Many well-known and accomplished pathologists consider themselves, above all, surgical pathologists. Conversely, there are those who asked, "If there were a surgical pathology (SP) board, then what is the anatomic pathology (AP) board?" "Would the AP board be a simplified version of the SP board, or would the SP board be a complex version of the AP board?" Furthermore, subspecialties such as dermatopathology and hematopathology have traditionally been part of anatomic/surgical pathology (and still are in many centers), so it seems illogical to reestablish the "parent" of these subspecialties, surgical pathology, as a parallel subspecialty. Some members objected to the regulatory constraints that would be required if surgical pathology fellowship programs were to be accredited and inspected, as would be necessary for board certification in surgical pathology.

Between the time this meeting was planned and when it was held, the American Board of Pathology eliminated the fifth (credentialing) year requirement. For some ADASP members, this act not only changed pathology training but also their perception of the desirability and necessity of a subspecialty board in SP. Specifically, the predictable yet major issue was raised: "Can the average resident competently practice surgical pathology upon completing a standard AP/CP (24 months of each) residency?" From the perspective of responsibility to the public, are our residents ready? Some expressed the problem as, "Can they be trusted to read my prostate or breast biopsy?" The majority of anatomic/surgical pathology directors have seemed skeptical, making the SP fellowship a near requirement, if a 4-year AP/CP training scheme were to be accepted.

This was the background to the ADASP meeting in February 2002. Representatives of the American Board of Pathology (ABP), the Residency Review Committee of the Accreditation Council on Graduate Medical Education (ACGME), Program Directors Section of the Association of Pathology Chairs, the College of American Pathologists, and 76 members of ADASP were present. The views of these major pathology organizations were presented by the spokespersons present. Drs. Richard Kempson and Mark Wick had agreed to speak, respectively, pro and con subspecialty certification in surgical pathology, but Dr. Wick changed his mind and spoke for a boarded subspecialty because the ABP had decreased 
the training time from 5 to 4 years. The barrage of questions and comments for all speakers were challenging and constructive.

Near the end of the meeting, several votes were taken among the membership present with regard to the major topics of discussion. There was a clear majority who voted against board certification in surgical pathology, but the wider accreditation of surgical pathology fellowships by ACGME was overwhelmingly favored. Most of those present voted to recommend a minimum of 36 months training in AP, and a clear majority voted against giving credit toward board eligibility for 12 months of research, although the situation was somewhat less clear with regard to credit for post-pathology course fellowships.

In view of the great importance that ADASP accords to training in AP and to ensure the broadest possible input, a formal postal ballot of the entire ADASP membership was undertaken after the meeting. The results were shared with the ABP at a meeting of the Cooperating Societies of the ABP, held in Chicago in May. The poll indicated that $82 \%$ (of $>90$ respondents) think that a minimum of 3 years of training in AP is required. If $\mathrm{AP}$ training remains at 2 years (which ADASP sincerely hopes is not the case), then $98 \%$ of voting ADASP members think that no credit should be allowed for research experience and $85 \%$ think that no credit should be allowed for a post-sophomore fellowship. The majorities were smaller in the context of a 3-year minimum training period, but most voting ADASP members still thought that no credit should be given for a year of research, whereas most thought that credit should be allowed for a post-sophomore fellowship within a 3-year training period.

A variety of additional pragmatic possibilities to address the need for adequate training have been suggested, at least one goal of which would be to maintain at least some flexibility to accommodate the needs of the research community. One possibility, which has considerable potential and also appears to have some support from the College of American Pathologists, would be to establish a separate 4-year academic track to board certification, in which a year's credit for research would only be given for those doing straight $\mathrm{AP}$ or straight $\mathrm{CP}$. Another suggestion, put forward by Dr. Jack Frable, in the context of a 5-year program, would be for all pathology residents to start out with 12 months each of AP and $\mathrm{CP}$, to be followed by an additional 12 months of core training in either AP or CP (depending on career preference), followed by 24 months of either straight AP subspecialties (including potentially cytopathology) or 24 months of CP subspecialties.

The principal conclusion of this important meeting and the subsequent ballot is that the ADASP membership believe strongly that a minimum period of 36 months of training in AP is required to be competent in the specialized practice environment of modern pathology. If total training in $\mathrm{AP} / \mathrm{CP}$ (the usual track for most trainees) is going to be reduced to 4 years, then research experience should not be substituted for diagnostic training, especially if subsequent board certification is going to indicate clinical competence. Separate board certification in surgical pathology was clearly not favored, but there is substantial and increasing support for the idea of ACGME accreditation of surgical pathology fellowships.

M. Timothy Smith, M.D.

Department of Pathology

Medical University of South Carolina

Charleston, South Carolina, U.S.A.

James L. Connolly, M.D.

Department of Pathology

Beth-Israel Deaconess Medical Center

Boston, Massachusetts, U.S.A.

Christopher D. M. Fletcher, M.D., F.R.C.Path.

Department of Pathology

Brigham and Women's Hospital

Boston, Massachusetts, U.S.A.

On Behalf of the Association of Directors of Anatomic and Surgical Pathology

\section{Risk for Subsequent Development of Breast Cancer}

\section{To the Editor:}

We read with interest the recent article by Shaaban et al., in which the risk for subsequent development of breast cancer was assessed for various benign lesions of the breast. ${ }^{17}$ The study population consisted of 120 study cases and 382 control cases. It is important to take the opportunity to emphasize several considerations that must be kept in mind because the statistical data may potentially misguide readers. Also, detailed comments regarding blunt duct adenosis (BDA) are warranted.

Before addressing specific topics, there are several general issues of note. On page 422 (in the first sentence of the last paragraph of the introduction), the authors state that they have studied high-risk lesions; however, none of the lesions studied is considered high risk, certainly not by standards in the United States. ${ }^{4}$ The authors stated that the case was reviewed by two pathologists to establish a consensus diagnosis, but it was not stated what proportion of diagnoses were changed after con- 
sensus readings. In assessment of relative risks (RRs), Shaaban et al. ${ }^{17}$ accepted the development of not only invasive carcinoma but also subsequent in situ carcinoma, and it is not stated what proportion were in situ or invasive. Major studies ${ }^{5,7,15}$ that have assessed the RR for various "benign" lesions and have served as the basis for follow-up recommendations have used only invasive carcinoma (not in situ carcinoma) as an end point. Because some studies do not even specify whether their end points included invasive carcinoma only versus invasive and in situ carcinomas, we think that it is more important to only include patients with invasive carcinoma as an end point. Therefore, the RR is in effect exaggerated by inclusion of ductal carcinoma in situ (DCIS) and lobular carcinoma in situ as end points. Further confounding the issue and despite inclusion of ductal and lobular in situ carcinomas, the authors chose to exclude low-grade DCIS from their end point. Because atypical ductal hyperplasia $(\mathrm{ADH})$ generally progresses to low-grade DCIS and not high-grade DCIS, we wonder what justifies this twisted exclusion/inclusion approach. Although they state which criteria they used for diagnosing $\mathrm{ADH},{ }^{16}$ they do not necessarily state that they are using criteria from the same reference ${ }^{16}$ for defining what threshold they used in diagnosing low-grade DCIS and how they separated low-grade DCIS from ADH: was one space with complete cribriform or micropapillary pattern of involvement sufficient? The impact of these inclusions/exclusions on the RR could be substantial. More significantly, these manipulations make the validity of the RR obtained in their study questionable and its comparison with the RRs obtained in other studies pointless.

In the breast, atypical lesions are obviously of particular concern as a risk factor for subsequent development of invasive carcinoma. We find it troublesome that the authors fail to provide any illustrations for atypical lobular hyperplasia (ALH), the lesion with the highest RR of 4.55 in their study, even though they have profusely illustrated lesions of lesser significance. Given the wellknown variation in criteria and/or application of the same criteria for atypical lesions, illustration of the lesion is the only way the reader could determine the type of lesion assessed in the study.

In this study, the atypical lesions (ALH and ADH) accounted for only 17 and 18 cases, respectively. They were obtained from two medical centers over a span of 20 years when the extent of surgical excision and sampling of the biopsies would have changed significantly. The numbers reflect a very limited experience with these lesions, even if only one individual were responsible for the performance of the entire task. These numbers are too small to provide any meaningful analysis, and a vastly larger sample size is needed if we are to be convinced of RRs that are assigned to breast lesions. For example, in the study by Page et al., the RR was determined based on an analysis of 150 and 126 cases of ADH and ALH, respectively. ${ }^{15}$ Interestingly, the mean follow-up period for the Shaaban et al. study is 66.95 months, ${ }^{17}$ which is too short for ALH, the lesion with the highest RR in this study.

The confidence intervals for the RR with ALH and ADH were 1.77-11.69 and 0.80-5.11, respectively, another issue of concern. ${ }^{17}$ Not only are the confidence intervals quite broad, but the lower limit of these confidence intervals is very close to 1.0 , further casting doubt on the strength of the statistical associations.

Regarding ADH, analysis of the RR is important because it allows comparison between at-risk and reference populations. Interestingly, despite inclusion of in situ carcinomas as an end point, the RR associated with ADH in the study is somewhat low (2.03) ${ }^{17}$ and differs substantially from the previously established RR of about 5 for ADH based on acceptance of only invasive carcinoma as the end point. ${ }^{4}$ The risk with ADH by Shaaban et al. was only slightly increased compared with the RR of 1.53 associated with hyperplasia of the usual type and even lower than the RR of 2.39 associated with solitary papilloma in their study. ${ }^{17}$ The authors did not find a statistically significant difference for the risk with ADH among the study and control cases $(\mathrm{p}=0.129),{ }^{17}$ but the percentage of ADH was far lower in the control group (5.83\% for study group vs. $2.87 \%$ for the control group). It is not stated clearly in Materials and Methods whether core biopsies were also included. Obviously, this would have a very critical effect on the data. Although this risk for the subsequent development of carcinoma in their study may seem small, it is definitely known that the chance of having carcinoma present in an excisional biopsy following a diagnosis of ADH on a core biopsy is quite substantial. ${ }^{6,9-11,13}$

Although Azzopardi provided fine descriptions and illustrations of $\mathrm{BDA},{ }^{2}$ standardization in the literature for this term does not exist, and lesions with varying histologic appearances have been illustrated as BDA in different authoritative textbooks. ${ }^{8,14}$ The illustrations in Figures 1 to 3 by Shaaban et al. ${ }^{17}$ for various types of BDA appear to be minimally altered lobules, and they do not exhibit a recognizable form of adenosis. Why use the term BDA for minimally altered lobules, some of which do not even show an increase in the number of acini (Figure 3 of Shaaban et al. ${ }^{17}$ )? In fact, the "BDA" illustrated in Figure 1 (of Shaaban et al. ${ }^{17}$ ) is remarkably similar to what Azzopardi has illustrated as "cystic lobular involution." "Shaaban et al. have made an admirable attempt to stratify BDA into different subcategories and assign RRs for each despite the small number of cases in the most important category of BDA: atypical columnar cell metaplasia (BDA-ACCM) ${ }^{17}$ Unfortunately, a high 
magnification illustration for Figure 4A (of Shaaban et al. ${ }^{17}$ ) (BDA-ACCM) is not available, and the illustration of the intermediate magnification illustration of BDAACCM in Figure 4B (of Shaaban et al. ${ }^{17}$ ) looks suspicious for pagetoid growth of lobular intraepithelial neoplasia (ALH or lobular carcinoma in situ). Although their category of BDA-columnar cell metaplasia did not have an increased RR, we find it interesting that their "BDAACCM" and "BDA, not otherwise specified" (BDANOS) had slightly increased RRs (2.32 and 2.08, respectively), although the lower limit of the confidence intervals is <1.0. Curiously, the RR for BDA-ADH was only 1.59 , which would almost imply that $\mathrm{ADH}$ is protective when present with BDA and that ACCM is more ominous than ADH.

The authors do not clearly state whether or not other lesions were concurrently present in biopsies harboring BDA. It is unlikely that BDA was the only lesion present in such samples. Alternatively, they also do not state how often BDA was found among their ALH cases. We do agree, however, that their BDA belongs to the interesting broad spectrum of columnar cell lesions. Although Shaaban et al. mentioned some of the several designations used in the literature for this lesion, ${ }^{17}$ they failed to mention others such as "clinging carcinoma" 3 and "flat epithelial atypia," nor did they cite molecular studies on the atypical variants. ${ }^{12}$

This brings us to an extremely important question: are columnar cell lesions an early form of "atypia?" What Shaaban et al. illustrate as BDA-ACCM ${ }^{17}$ is certainly not particularly worrisome. At the Armed Forces Institute of Pathology, the term "flat epithelial atypia" is used for lesions characterized by monotony and mild to moderate atypia. We avoid the term columnar cell metaplasia because it promotes an inaccurate use of the term metaplasia: a change in cell shape is not a metaplasia. Also, many mammary epithelial cells, including apocrine cells and many secretory cells, are also columnar. The frequent finding of flat epithelial atypia in ducts that harbor low-grade intraepithelial carcinomas and their common association with invasive tubular carcinoma point to a definite potential for progression of these lesions to invasive carcinomas. Also supportive of this idea is that molecular studies have shown alterations in flat atypias similar to those reported in invasive ductal carcinomas whether the flat atypia is present as the only lesion or next to an intraepithelial or invasive carcinoma. ${ }^{12}$ The findings in the study by Shaaban et al. ${ }^{17}$ for some of the BDA lesions with possibly very limited atypia could provide clinical follow-up support, suggesting a potential for progression associated with some types of columnar cell lesions.

If the illustrations reflect the range of "atypia," including the most advanced atypia encountered in "columnar cell lesions" in the study, then at most a very limited number of their cases would qualify as what is designated as flat epithelial atypia at the Armed Forces Institute of Pathology that we get concerned about. We wonder if examples of higher-grade flat epithelial atypias or columnar cell lesions were seen by Shaaban et al. ${ }^{17}$ but rather classified as low-grade DCIS and, therefore, excluded from the study.

The authors state that the goal of the study was to define precise morphologic criteria that are associated with an increased risk of breast cancer. The findings of the study, however, did not result in a morphologic definition of the lesions examined.

It is often too easy to read articles assessing risk for breast cancer and become biased by only looking at the reported RR for the sake of brevity. It is hoped that readers and reviewers will approach data presented in subsequent similar papers with objective criticism and take all statistical factors into consideration: sample size, confidence intervals, and study design. Inclusion of an editorial comment would also help focus on the issues that should be of concern.

Finally, a well-organized study of pure flat epithelial atypias/columnar cell lesions with "clinging cytologic atypia" is needed to more appropriately address the clinical significance of the gradations within this perplexing category of lesions.

Russell Vang, M.D.

Fattaneh A. Tavassoli, M.D.

Department of Gynecologic \& Breast Pathology

Armed Forces Institute of Pathology

Washington, DC, U.S.A.

\section{REFERENCES}

1. Azzopardi JG. Nomenclature of the microanatomy of the breast: parts affected in different diseases: normal structure and involution. In: Major Problems in Pathology, vol. 11. Problems in Breast Pathology. Philadelphia: Saunders, 1979:17-20.

2. Azzopardi JG. Terminology of benign diseases and the benign epithelial hyperplasia. In: Major Problems in Pathology, vol. 11. Problems in Breast Pathology. Philadelphia: Saunders, 1979:25-32.

3. Azzopardi JG. Underdiagnosis of malignancy. In: Major Problems in Pathology, vol. 11. Problems in Breast Pathology. Philadelphia: Saunders, 1979:193-203.

4. Cancer Committee of the College of American Pathologists. Is 'fibrocystic disease' of the breast precancerous? Consensus meeting, October 3 to 5, 1985, New York. Arch Pathol Lab Med 1986; 110:171-3.

5. Carter CL, Corle DK, Micozzi MS, et al. A prospective study of the development of breast cancer in 16,692 women with benign breast disease. Am J Epidemiol 1988;128:467-77.

6. Dahlstrom JE, Sutton S, Jain S. Histological precision of stereotactic core biopsy in diagnosis of malignant and premalignant breast lesions. Histopathology 1996;28:537-41.

7. Dupont WD, Parl FF, Hartmann WH, et al. Breast cancer risk 
associated with proliferative breast disease and atypical hyperplasia. Cancer 1993;71:1258-65.

8. Ellis IO, Elston CW, Goulding H. Fibrocystic change. In: Elston CW, Ellis IO, eds. Systemic Pathology, vol. 13. The Breast, 3rd ed. New York: Churchill Livingstone, 2000:55-7.

9. Ioffe OB, Berg WA, Silverberg SG, et al. Mammographichistopathologic correlation of large-core needle biopsies of the breast. Mod Pathol 1998;11:721-7.

10. Jackman RJ, Nowels KW, Shepard MJ, et al. Stereotaxic largecore needle biopsy of 450 nonpalpable breast lesions with surgical correlation in lesions with cancer or atypical hyperplasia. Radiology 1994;193:91-5.

11. Liberman L, Cohen MA, Dershaw DD, et al. Atypical ductal hyperplasia diagnosed at stereotaxic core biopsy of breast lesions: an indication for surgical biopsy. Am J Roentgenol 1995;164:1111-3.

12. Moinfar F, Man YG, Bratthauer GL, et al. Genetic abnormalities in mammary ductal intraepithelial neoplasia-flat type ('clinging ductal carcinoma in situ'): a simulator of normal mammary epithelium. Cancer 2000;88:2072-81.

13. Renshaw AA, Cartagena N, Schenkman RH, et al. Atypical ductal hyperplasia in breast core needle biopsies: correlation of size of the lesion, complete removal of the lesion, and the incidence of carcinoma in follow-up biopsies. Am J Clin Pathol 2001;116:92-6.

14. Rosen PP. Adenosis and microglandular adenosis. In: Rosen's Breast Pathology, 2nd ed. Philadelphia: Lippincott Williams \& Wilkins, 2001:149.

15. Page DL, Dupont WD, Rogers LW, et al. Atypical hyperplastic lesions of the female breast: a long-term follow-up study. Cancer 1985;55:2698-708.

16. Page DL, Rogers LW. Combined histologic and cytologic criteria for the diagnosis of mammary atypical ductal hyperplasia. Hum Pathol 1992;23:1095-7.

17. Shaaban AM, Sloane JP, West CR, et al. Histopathologic types of benign breast lesions and the risk of breast cancer: case-control study. Am J Surg Pathol 2002;26:421-30.

\section{Authors' Reply:}

In response to the letter by Vang and Tavassoli, subsequent to our earlier paper, ${ }^{28}$ we appreciate the important points raised. There are some issues related to the understanding of our data that we would like to address. In our study, we sought primarily to examine morphologic lesions associated with subsequent breast cancer development by examining the frequencies of occurrence of benign breast lesions in study cases (patients who underwent biopsies revealing benign breast lesions and thereafter developed breast cancer) and matched controls (patients who had benign breast biopsies but did not develop breast cancer). Patients who subsequently developed breast cancer could be classified retrospectively as "high risk." However, we did not follow the U.S. standards for defining such lesions, ${ }^{13}$ nor did we follow the Gail mathematical model to predict breast cancer risk. ${ }^{12}$ Thus, use of the term "high risk" in the introduction refers to lesions occurring in patients that definitely progressed to develop breast cancer and are, therefore, defined biologically to be of potential "high risk."

To maximize consistency, all lesions were reviewed by two pathologists with special expertise in breast pathology. Inconsistency occurred on diagnosing some of the examples of atypical ductal hyperplasia (ADH) versus well-differentiated ductal carcinoma in situ (DCIS). However, this was resolved by joint revision of slides and a consensus was reached. Several studies have highlighted the problem of diagnostic inconsistency in the diagnosis of proliferative breast lesions, even among expert pathologists, with agreements for proliferative lesions with atypia ranging from fair to poor. ${ }^{8,24,25,33}$ In the largest study to date, 51 pathologists were circulated with sets of 12 slides, each at six monthly intervals for 3 years, ${ }^{30}$ to assess the morphology. Consistent with previous reports, this study showed poor agreement on diagnosing ADH (kappa $=0.25$ ). The development and use of standardized criteria for diagnosing ADH have been shown to maximize consistency. ${ }^{27}$

The end point in our study was development of breast carcinoma, irrespective of whether in situ or invasive. Of 120 subsequent carcinomas, only 11 were pure DCIS. We think that inclusion of DCIS as the end point is appropriate because certain benign proliferative breast lesions are likely to progress to low-grade DCIS. Exclusion of the latter lesion as an end point is likely to underestimate the risk associated with those benign proliferative lesions. Furthermore, defining morphologic lesions that are associated with increased risk for developing in situ as well as invasive cancer was another aim of our study. Interestingly, the proportion of pure DCIS cases in the population of subsequently developed cancers was small $(9.16 \%)$ such that it would not compromise comparison with other studies that used the sole development of invasive carcinoma as the end point.

We emphasize that low-grade DCIS was excluded only from the benign lesions of both cases and controls and not from the subsequently developed carcinomas. Indeed, pure in situ carcinomas of the latter included four examples of low-grade DCIS. Lesions diagnosed as lowgrade DCIS within the original study population were excluded from both cases and controls because they represent malignant examples at the initial diagnoses that were, by definition, an exclusion criterion. Six of 15 malignant examples excluded from study lesions were classified as low-grade DCIS. Similarly, lobular carcinoma in situ was excluded from the original case-control study in a single patient. To diagnose low-grade DCIS, as defined in the article, we used the morphologic criteria of Page and Rogers. ${ }^{22}$ The size of lesions was also incorporated to separate ADH from low-grade DCIS. ${ }^{31} \mathrm{Be}$ cause DCIS was excluded only from benign lesions, we think that it was perfectly acceptable to include this as a distinct category within the malignancies that developed subsequently.

We chose to provide illustrations for the different morphologic features of blunt duct adenosis, including those with $\mathrm{ADH}$, because it is an area of controversy both at the level of terminology and morphology. Because we attempted to provide a new classification for this interesting group of lesions, based on morphologic features as well to identify the associated level of breast cancer risk 
associated with each subtype, it was mandatory to illustrate how we identified each category. As explained in Materials and Methods, we followed the guidelines of the U.K. National Coordinating Group for Breast Cancer Screening Pathology ${ }^{20}$ for diagnosing benign lesions, including atypical lobular hyperplasia (ALH). As pathologists are aware of the standard criteria for diagnosing such lesions, further illustrations were not considered necessary.

In this study, atypical lesions (ALH and ADH) occurred in 35 of 583 patients $(6.01 \%)$. It is to be noted that this number reflects examples of atypical hyperplasia within the population of 382 cases and controls and not within the total number of patients received in the medical centers of study spanning a 20-year period. We do not regard this percentage as small because it is higher than that reported in the study by Page et al. (3.6\%). ${ }^{23} \mathrm{~A}$ larger number of ADH and ALH examples inevitably occurred within the total number of 17,105 breast biopsies received at the centers of study (Royal Liverpool University Hospital, Broadgreen Hospital, and Lourdes Hospital). The designation of our study was based on matching every study case for age and date of biopsy with three control patients. A total of 580 fulfilled those stringent selection criteria. However, further exclusion of inadequate and malignant lesions at the initial diagnoses reduced the total number to 502 patients. Nevertheless, the total number of biopsies included in the study was even larger (674) because we also examined repeat biopsies from both groups.

The mean follow-up period in our study was 66.95 months. The total period of follow-up, however, was 20 years. For statistical analysis, the end point for cases was the development of breast cancer, whereas that for controls was the end of the follow-up period (end of January 1999). Because the majority of our cases progressed to breast cancer within the first 5 years following their first benign biopsies, the mean follow-up period was relatively short. The increased risk of breast cancer was previously shown to be greatest in the first 10-15 years following a benign biopsy showing atypical hyperplasia. ${ }^{6}$

Although the level of risk associated with $\mathrm{ADH}$ in our study was lower at 2.03 (confidence interval [CI] 0.85.11) than that previously reported, ${ }^{23}$ other studies have shown similar values. Marshall et al. showed an odds ratio of 2.4 (CI 1.3-4.5) for $\mathrm{ADH} .{ }^{17}$ Bodian et al. ${ }^{2}$ reported a relative risk of 2.3 (CI 1.6-3.4) and 3.0 (CI 1.5-6.0) for hyperplasia with mild atypia and hyperplasia with moderate or severe atypia, respectively. McDivitt et al. ${ }^{18}$ showed that women with hyperplasia with atypia had an odds ratio of 2.6 (CI 1.6-4.1) for subsequently developing breast carcinoma. Other investigators have combined both ADH and ALH and provided an overall level of risk for atypical hyperplasia, 3.7 (CI 2.1-6.6), ${ }^{16}$
5.7 (CI 3.0-10.6), ${ }^{4} 5.3$ (CI 3.1-8.8), ${ }^{5} 4.3$ times the breast cancer risk of women without proliferative disease (CI 1.7-11), ${ }^{7}$ and 3.8 (CI: 2.4 to 5.9). ${ }^{15}$ A single study reported a very high risk of developing breast cancer in women with atypical hyperplasia (odds ratio 13.0, CI 4.1-41.7). ${ }^{24}$ Providing an overall relative risk for both $\mathrm{ADH}$ and ALH might explain, at least in part, the discrepancies in the levels of risk reported by different investigators. The $\mathrm{p}$ value of 0.129 in our study quoted by the authors reflects the statistical significance of frequency of $\mathrm{ADH}$ occurrence between cases and controls and not differences in relative risk between both groups (see Table 3 of original paper ${ }^{28}$ ). Although the lesion occurred more frequently in patients who subsequently developed breast cancer when compared with control subjects $(5.83 \%$ vs. $2.87 \%$ respectively), this was not statistically significant, probably because of the relatively limited number of lesions included.

As we explained in Materials and Methods, where a patient had more than one benign biopsy, then all specimens were examined. Core biopsies were therefore examined. Where sufficient breast tissue was available for histopathologic assessment, then core biopsies were included; otherwise, biopsies were deemed inadequate and were excluded from study cases and controls. In no instances was ADH diagnosed solely on a core biopsy.

We agree with the authors on the lack of standardization of terms describing cystically dilated breast lobules with and without atypia. We think that BDA encompasses a spectrum of morphologic changes that, in its very minimal forms, might resemble cystic involution of lobules. Nevertheless, BDA exhibits a larger number of acini within a cellular rather than fibrous stroma and is often surrounded by pseudoangiomatous stromal hyperplasia. The acini in BDA may undergo some dilatation to form microcysts. When this change is prominent, particularly when associated with apocrine snouts, the picture resembles apocrine cysts. We preferred to use the term BDA, as coined by Foote and Stewart ${ }^{10}$ and used by Azzopardi, ${ }^{1}$ because lesions represent a form of organoid lobular hypertrophy similar to that illustrated by Azzopardi. ${ }^{1}$ Although the term "columnar cell metaplasia" might not be absolutely correct in the sense that it does not represent a true metaplastic change, the term is so widely used among pathologists to describe the morphologic features of dilated lobules with tall columnar lining that the introduction of different designations will be a source of confusion. Cells appear strikingly columnar in comparison with epithelial lining of normal breast. Columnar metaplasia, ${ }^{3}$ columnar alteration of lobules, ${ }^{21}$ columnar cell hyperplasia, ${ }^{26}$ and columnar alteration with prominent apical snouts and secretions ${ }^{11}$ were all used to describe those lesions. The use of standardized terms will help maximize consistency in the 
diagnosis of this interesting but conflicting area of breast pathology.

Care was taken to show all photomicrographs for BDA categories at identical magnifications to avoid bias caused by inconsistency in illustrating the size of lesions and constituent cells. Although the relative risk associated with some BDA subtypes (BDA-NOS, BDA+Ca ${ }^{++}$, $\mathrm{BDA}+\mathrm{ACCM})$ was high $(2.08,1.75$, and 2.32 , respectively), the confidence interval for the important category of BDA+ACCM covered 1.0 (0.95-5.62). The broad confidence intervals associated with the relative risk of some benign categories in our study reflect small numbers of positive cases.

We recognize that the lack of statistical significance might result from the small number of examples included in each subcategory caused by stratification of this group into six subtypes. Therefore, further large studies with long follow-up periods are needed to confirm our findings.

We have indeed stated that combination of lesions occurred in our cohort and that combined lesions were recorded separately (original paper, ${ }^{28}$ p. 426 , comment below Table 3). Examples of BDA coexisted with other benign categories, including ALH. BDA-NOS was found in three biopsies containing $\mathrm{ALH}$. BDA+Ca ${ }^{++}$coexisted with ALH in three biopsies, whereas ALH occurred concomitantly with BDA+HUT in two instances and with $\mathrm{BDA}+\mathrm{CCM}$ in seven cases. In none of the biopsies was ALH combined with either BDA+ADH or $\mathrm{BDA}+\mathrm{ACCM}$.

We acknowledge the differences in terminologies used in the literature to describe BDA lesions. In our article, we quoted terminologies that relate to the classification we used in the current work. "Clinging carcinoma" was not mentioned because we regard it as a form of DCIS that was excluded from the benign study population. Furthermore, various criteria are used by different authors to diagnose clinging carcinoma. ${ }^{1,9,32}$ Some further subdivide clinging DCIS into monomorphic and polymorphic variants. ${ }^{14}$ The monomorphic variant has mild nuclear atypia and thus might be missed on low power examination. The term "flat epithelial atypia," although widely used at the Armed Forces Institute of Pathology, is seldom used in the United Kingdom.

We are aware of the molecular study by Moinfar et al., ${ }^{19}$ in which the authors studied loss of heterozygosity on 22 cases with mammary ductal intraepithlial neoplasia (DIN) flat-type of both variants using eight polymorphic DNA markers with a higher rate of $\mathrm{LOH}$ in classic-type DCIS. The flat lesion in 17 of $22(77 \%)$ showed LOH at a minimum of 1 locus (monomorphic $70 \%$ and polymorphic $89 \%$ ), with the locus 11q21-q23.2 containing the highest level of $\mathrm{LOH}$. The results illustrate that these lesions share the same genetic alter- ations identified in adjacent in situ and welldifferentiated infiltrating ductal carcinoma, ${ }^{29}$ providing further evidence of links between atypical flat lesions and both in situ and invasive carcinoma. Recently, Simpson et al. ${ }^{29}$ studied 42 lesions of columnar alteration with prominent apical snouts and secretions (CAPSS) and flat epithelial atypia (FEA) (17 cases) using immunohistochemistry and comparative genomic hybridization. Comparative genomic hybridization data from 17 microdissected lesions identified genetic alterations previously seen in DCIS/invasive carcinoma $(1 \mathrm{q}+, 13 \mathrm{q}-, 17 \mathrm{q}+, 20 \mathrm{q}+)$ with some lesions showing common changes in associated malignant lesions. The authors concluded that CAPSS/FEA is a precursor lesion for progression to ADH/in situ malignancy. ${ }^{29}$ These molecular findings undoubtedly add to our understanding of the biology of these lesions and their possible relationship to mammary carcinogenesis. However, detailed analysis of the available molecular data of these lesions was beyond the scope of our paper.

In our work, a single lining of mild to moderately atypical epithelium would be included in the category of $\mathrm{BDA}+\mathrm{ACCM}$. However, higher grades of atypia were regarded as low-grade DCIS and therefore excluded from the initial study population.

In the current study, we confirmed the previously reported elevated levels of risk associated with some benign proliferative breast lesions, and we have proposed a morphologic classification for the group of cystically dilated breast lobules both with and without atypia. We also identified the level or risk associated with each of the six subcategories. By doing so, we attempted to achieve, at least in part, the primary goal of this study of identifying morphologic features of benign lesions associated with increased breast cancer risk.

In our paper, ${ }^{28}$ we emphasized, in more than one instance in the Discussion, the limitations in sample size regarding different benign categories, including ADH (p. 427, last paragraph), BDA subtypes (p. 429, first paragraph), and the need for further large-scale studies (p. 427, second paragraph, and p. 429, last paragraph). We also explained the lack of statistical significance reflected by confidence intervals covering 1 (p. 427, last paragraph), although associated with high relative risk. The table in the Discussion (p. 429) was presented to help readers compare the current results with data from the literature, taking into account differences in sample size, length of follow-up, etc., and to avoid bias caused by failure to consider those factors on reading our results. We think that further large studies with outcome data are invaluable to confirm the putative role of this group of lesions in mammary tumorigenesis. 
Abeer Shaaban, M.B., B.Ch., M.Sc.

Christopher S. Foster, M.D., Ph.D., D.Sc., F.R.C.Path.

Department of Cellular and Molecular Pathology

University of Liverpool

Liverpool, U.K.

\section{REFERENCES}

1. Azzopardi JC. Problems in Breast Pathology. London: Saunders, 1979.

2. Bodian CA, Perzin KH, Lattes R, et al. Prognostic significance of benign proliferative breast disease. Cancer 1993;71:3896-907.

3. Bosner GM, Dossett JA, Jull W. Human and Experimental Breast Cancer. London: Pitman, 1961.

4. Carter CL, Corle DK, Micozzi MS, et al. A prospective study of the development of breast cancer in 16,692 women with benign breast disease. Am J Epidemiol 1988;128:467-77.

5. Dupont WD, Page DL. Risk factors for breast cancer in women with proliferative breast disease. N Engl J Med 1985;312:146-51.

6. Dupont WD, Page DL. Relative risk of breast cancer varies with time since diagnosis of atypical hyperplasia. Hum Pathol 1989;20: 723-5.

7. Dupont WD, Parl FF, Hartmann WH, et al. Breast cancer risk associated with proliferative breast disease and atypical hyperplasia. Cancer 1993;71:1258-65.

8. Elston CW, Sloane JP, Amendoeira I, et al. Causes of inconsistency in diagnosing and classifying intraductal proliferations of the breast: European Commission Working Group on Breast Screening Pathology. Eur J Cancer 2000;36:1769-72.

9. Eusebi V, Foschini MP, Cook MG, et al. Long-term follow-up of in situ carcinoma of the breast with special emphasis on clinging carcinoma. Semin Diagn Pathol 1989;6:165-73.

10. Foote FW, Stewart FW. Comparative studies of cancerous versus non-cancerous breast: basic morphological characteristics. Ann Surg 1945;121:6-35.

11. Fraser JL, Raza S, Chorny K, et al. Columnar alteration with prominent apical snouts and secretions: a spectrum of changes frequently present in breast biopsies performed for microcalcifications. Am J Surg Pathol 1998;22:1521-7.

12. Gail MH, Brinton LA, Byar DP, et al. Projecting individualized probabilities of developing breast cancer for white females who are being examined annually. J Natl Cancer Inst 1989; 81:1879-86.

13. Godfrey SE. Is fibrocystic disease of the breast precancerous? Arch Pathol Lab Med 1986;110:991.

14. Holland R, Peterse JL, Millis RR, et al. Ductal carcinoma in situ: a proposal for a new classification. Semin Diagn Pathol 1994;11: 167-80.

15. Jacobs TW, Byrne C, Colditz G, et al. Radial scars in benign breast-biopsy specimens and the risk of breast cancer. $N$ Engl J Med 1999;340:430-6.

16. London SJ, Connolly JL, Schnitt SJ, et al. A prospective study of benign breast disease and the risk of breast cancer. JAMA 1992; 267:941-4.

17. Marshall LM, Hunter DJ, Connolly JL, et al. Risk of breast cancer associated with atypical hyperplasia of lobular and ductal types. Cancer Epidemiol Biomarkers Prev 1997;6:297-301.

18. McDivitt RW, Stevens JA, Lee NC, et al. Histologic types of benign breast disease and the risk for breast cancer: the Cancer and Steroid Hormone Study Group. Cancer 1992;69:1408-14.

19. Moinfar F, Man YG, Bratthauer GL, et al. Genetic abnormalities in mammary ductal intraepithelial neoplasia-flat type ("clinging ductal carcinoma in situ'): a simulator of normal mammary epithelium. Cancer 2000;88:2072-81.

20. National Coordinating Group for Breast Screening Pathology. Pathology Reporting in Breast Cancer Screening (NHSBSP pub- lication no. 3). National Coordinating Group for Breast Screening Pathology, 1997.

21. Page DL, Anderson TJ. Diagnostic Histopathology of the Breast. Edinburgh: Churchill Livingstone, 1987.

22. Page DL, Rogers LW. Combined histologic and cytologic criteria for the diagnosis of mammary atypical ductal hyperplasia. Hum Pathol 1992;23:1095-7.

23. Page DL, Dupont WD, Rogers LW, et al. Atypical hyperplastic lesions of the female breast: a long-term follow-up study. Cancer 1985;55:2698-708.

24. Palli D, Rosselli del Turco M, Simoncini R, et al. Benign breast disease and breast cancer: a case-control study in a cohort in Italy. Int J Cancer 1991;47:703-6.

25. Rosai J. Borderline epithelial lesions of the breast. Am J Surg Pathol 1991;15:209-21.

26. Rosen PP. Rosen's Breast Pathology. Philadelphia: LippincottRaven, 1997.

27. Schnitt SJ, Connolly JL, Tavassoli FA, et al. Interobserver reproducibility in the diagnosis of ductal proliferative breast lesions using standardized criteria. Am J Surg Pathol 1992;16: 1133-43.

28. Shaaban AM, Sloane JP, West CR. Histopathologic types of benign breast lesions and the risk of breast cancer: case-control study. Am J Surg Pathol 2002;26:421-30.

29. Simpson PT, Gale T, Jones C, et al. Molecular genetic analysis of columnar cell hyperplasia/flat epithelial atypia lesions using comparative genomic hybridisation. J Pathol 2002; 198(suppl):7A.

30. Sloane JP, Ellman R, Anderson TJ, et al. Consistency of histopathological reporting of breast lesions detected by screening: findings of the U.K. National External Quality Assessment (EQA) Scheme. U.K. National Coordinating Group for Breast Screening Pathology. Eur J Cancer 1994;10:1414-9.

31. Tavassoli FA. Ductal carcinoma in situ: introduction of the concept of ductal intraepithelial neoplasia. Mod Pathol 1998;11: $140-54$.

32. Tavassoli FA. Pathology of the Breast. Norwalk, Connecticut: Appleton Lange, 1992.

33. Wells WA, Carney PA, Eliassen MS, et al. Statewide study of diagnostic agreement in breast pathology. J Natl Cancer Inst 1998; 90:142-5

\section{Cardiac Mucosal Changes in a Pediatric Population}

To the Editor:

I read with great interest the excellent article by Glickman et al. on their experience with cardiac mucosal changes in a pediatric population. ${ }^{4}$ The study agrees with our adult patient data in several important respects, as stated by the authors. ${ }^{1-3}$ These include the finding that increasing lengths of cardiac mucosa correlate with reflux ${ }^{1}$ and that the presence of eosinophils in cardiac mucosa correlates with reflux. ${ }^{3}$

I would like to address what the authors think are points of discordance between their data and our studies. My hope is to point out that there is little if any discordance. 
The first point of discordance stated by the authors is that they could not find pure oxyntic mucosa immediately distal to the squamous epithelium in any of their patients; this is different from our report in which $29 \%$ of patients had pure oxyntic mucosa immediately distal to the squamous epithelium. ${ }^{2}$ These two studies cannot reasonably be compared. The population reported by Glickman et al. is one that is a selective group that has a high probability of reflux disease. ${ }^{4}$ The case accrual rate is 8 to 9 per year with a $>20 \%$ prevalence of each of the following factors: vomiting, regurgitation, heartburn, dysphagia, H2 antagonist use, and proton pump inhibitor use. The rate of histologically active esophagitis in the squamous epithelium is $38 \%$, which is not much lower than the rate of histologic esophagitis in most studies of patients with proven reflux. Only seven patients in their study did not have upper gastrointestinal symptoms or histologic evidence of esophagitis. This cannot be compared with our normal autopsy population. What surprises me about Dr. Glickman's data is that $19 \%$ of the patients had no cardiac mucosa rather than the failure to find pure oxyntic mucosa. The prevalence of cardiac mucosa in an adult population with a high likelihood of reflux will be close to $100 \%$.

The second point of discordance relates to the authors' conclusion that the cardiac mucosa was found in the proximal stomach distal to the gastroesophageal junction (GEJ). This differs from our conclusion that cardiac mucosa is always esophageal and proximal to the GEJ. This discordance is one of interpretation, not data. Glickman et al. made all their measurements from the distal limit of the squamous epithelium. The conclusion that the mucosa immediately distal to the squamous epithelium is "proximal stomach" or "cardia" can only be true if the GEJ and squamo-columnar junction (SCJ) are coincidental. In fact, the authors state this to be true: "The gastroesophageal junction (GEJ) was defined endoscopically as the proximal limit of the gastric folds; in this study group it coincided with the distal limit of the squamouslined tubular esophagus. Thus biopsies that straddled the SCJ represented the distal esophagus and proximal stomach." This statement is a potential source of error because it assumes two things: 1) that the endoscopic data are absolutely reliable, and 2) that the endoscopist can recognize with absolute accuracy the difference between a coincident GEJ and SCJ and one in which the GEJ is separated from the SCJ by $1 \mathrm{~mm}$. If the endoscopist made a $1-\mathrm{mm}$ error, which is very feasible, then this causes a serious error in the interpretation of the data. It would mean that the cardiac mucosa restricted to the 1 $\mathrm{mm}$ distal to the squamous epithelium seen in $46 \%$ of Glickman et al.'s cases was proximal to the GEJ, that is, in the esophagus and not in the stomach. If the endoscopist made a 2-mm error, an even larger percentage of patients would not have cardiac mucosa in their stomach. It is my firm belief that millimetric lengths cannot be assessed accurately by anything other than microscopy, and to trust gross or endoscopic examination to delineate landmarks within 1 to $2 \mathrm{~mm}$ is a futile exercise fraught with error.

The authors appear to recognize this in their statement: "we suspect that longer lengths of pure mucous glands in the SCJ region represent proximal extension (metaplasia) into the distal esophagus." However, they cannot say this if they believed in the absolute coincidence of the GEJ and SCJ in their cases. For there to be proximal extension (cardiac metaplasia of squamous epithelium) into the distal esophagus, the GEJ must become separated from the SCJ by the proximally extending cardiac mucosa.

Measurements from the squamous epithelium can only determine the amount of cardiac mucosa present distal to the squamous epithelium. It is our contention that the true histologic GEJ is the proximal limit of oxyntic mucosa. The distance between this and the squamous epithelium represents the "proximal extension (metaplasia) of cardiac mucosa into the distal esophagus." In our practice, we think the most accurate and reproducible measurement in reflux disease is the distance between the first parietal cell and squamous epithelium. This is the length of pure cardiac mucosa and gives the most accurate histologic assessment of reflux disease. If this measurement is zero, the patient has no histologic evidence of reflux. As the distance increases from zero, the reflux-induced damage is greater. Initially, this is microscopic and not visualized endoscopically. The length at which it is visible endoscopically varies with the experience of the endoscopist, but it is probably in the 2- to 5-mm range at best.

Para Chandrasoma, M.D.

Department of Surgical Pathology

LAC+USC Medical Center

Los Angeles, California, U.S.A.

\section{REFERENCES}

1. Chandrasoma PT, LoKuherty DM, DeMeester TR, et al. Definition of histopathologic changes in gastroesophageal reflux disease. Am J Surg Pathol 2000;24:344-51.

2. Chandrasoma PT, Der R, Ma Y, et al. Histology of the gastroesophageal junction: an autopsy study. Am J Surg Pathol 2000;24: 402-9.

3. Der R, Tsao-Wei DD, DeMeester TR, et al. Carditis: a manifestation of gastroesophageal reflux disease. Am J Surg Pathol 2001; 25:245-52.

4. Glickman JN, Fox V, Antonioli DA, et al. Morphology of the cardia and significance of carditis in pediatric patients. Am J Surg Pathol 2002;26:1032-9. 
Authors' Reply:

The authors thank Dr. Chandrasoma for his interest and insightful comments regarding our study. ${ }^{7}$ The issues raised in his letter are important for our understanding of the pathophysiology of gastroesophageal reflux disease. As discussed in our paper, we think that our data support Dr. Chandrasoma's hypothesis that gastroesophageal reflux probably leads to an increase in the length of "cardia-type" mucosa, comprised of mucous glands, in the region of the gastroesophageal junction (GEJ). However, we do not agree with his additional argument that the mere presence of mucous glands at the level of the GEJ is necessarily always the result of metaplasia. We will address Dr. Chandrasoma's comments to clarify our position.

Dr. Chandrasoma correctly points out that our two studies differ with regard to the finding of pure oxyntictype mucosa at the squamocolumnar junction $(0 \%$ in our study ${ }^{7}$ vs. $29 \%$ in the study by Chandrasoma et $\mathrm{al}^{2}$ ). As indicated in our Discussion, we also agree that the results of these two studies are difficult to compare because they include different patient populations with different methods of tissue sampling (autopsy vs. biopsy). However, although our patient population does have a relatively high proportion of individuals with active esophagitis and/or reflux symptoms (up to $38 \%$ ), we would also like to emphasize that reflux disease is often asymptomatic, and, thus, even a normal autopsy population would be expected to include a significant percentage (up to $21 \%$ ) of individuals with this disorder. ${ }^{11,12}$ In our study, unlike that of Chandrasoma et al., ${ }^{1,2}$ we correlated the histologic findings with clinical information. Importantly, in our population of patients who were known to be completely free of reflux symptoms and active esophagitis, we still did not find even one individual who had pure oxyntic mucosa. This finding has been shown by others as well ${ }^{9}$ and supports our contention that the mere presence of cardiatype mucosa does not always imply reflux disease. Finally, Dr. Chandrasoma commented that $19 \%$ of the patients in our study "had no cardiac mucosa" at the squamocolumnar junction. In fact, they had a mixture of mucous and oxyntic-type glands in the cardia region, which has been reported before as a histologic variation of the anatomic cardia region, even by Dr. Chandrasoma's group. ${ }^{1}$

Regarding the "location" (esophageal vs. gastric) of cardiatype mucosa, we agree entirely with Dr. Chandrasoma that this distinction is dependent upon careful endoscopic correlation and that this technique may not be $100 \%$ reliable in determining the site of origin of a particular biopsy from the GEJ region. However, the patients in our study were examined by highly experienced endoscopists, and any patients with confusing or equivocal endoscopic findings were excluded from the study. Furthermore, although we agree that a distance of 1-2 $\mathrm{mm}$ may be difficult to assess accurately at endoscopy, we would also like to point out that, in the same vein, it is also equally likely that any particular biopsy from an apparently normal-appearing squamocolumnar junction (present at the proximal level of the GEJ) may have actually been obtained from the proximal stomach, and not just the distal esophagus, as indicated by Dr Chandrasoma. Thus, as indicated in the discussion of our paper, we cannot be completely certain that the presence of cardia-type mucosa in the GEJ region represents mucosa proximal (esophageal) or distal (gastric) to the true GEJ. Therefore, we think that Dr. Chandrasoma's conclusion that "cardiac mucosa is always esophageal" is equally unjustifiable based on the same argument. Finally, we think that cardiatype epithelium can occur in the proximal stomach because this has been shown repeatedly in studies by both our group and by those of others that have identified carditis (in epithelium comprised of mucous glands) in the setting of Helicobacter pylori gastritis. $3,8,10,14$

As stated above, we agree with the author that one cannot be certain that longer lengths of pure mucous glands always represent proximal extension of cardia-type mucosa into the esophagus. We prefer to remain open to the possibility that the "normal" length of the cardia is variable, as previously shown by others. $^{9}$

Finally, we agree with Dr. Chandrasoma's statement that "measurements from the squamous epithelium can only determine the amount of cardiac mucosa distal to the squamous epithelium." However, based on the results of our studies and those of others, we do not concur with the statement that "the true histologic GEJ is the proximal limit of oxyntic mucosa." As pointed out by Dr. Chandrasoma, this is only their "contention," and is an arbitrary one at that, having never been proven in a controlled study by either their group or others. We appreciate the findings by Chandrasoma et al. that the degree of reflux correlates with the length of cardia-type mucosa at the GEJ, ${ }^{1,4}$ but we would like to emphasize that their conclusions are based on studies without a symptom-free control group. In addition, we and others have reported a significant number of patients, both adult and pediatric, who have inflammation and $H$. pylori organisms in cardia-type mucosa comprised of mucous glands and who also showed an endoscopically normal GEJ with a straight squamocolumnar junction and an absence of reflux symptoms. ${ }^{3,5,8,14,15}$ This observation provides further support for the notion that cardiatype mucosa can be found in the proximal stomach and is not always necessarily the result of reflux. Finally, we have found that patients with reflux frequently have multilayered epithelium, which appears to be an early pre- 
cursor of intestinal metaplasia., ${ }^{6,13}$ We have never observed this type of epithelium in $H$. pylori-associated carditis. $^{16}$

Jonathan N. Glickman, M.D., Ph.D.

Robert D. Odze, M.D.

Brigham and Women's Hospital

Harvard Medical School

Boston, Massachusetts, U.S.A.

\section{REFERENCES}

1. Chandrasoma PT, Lokuhetty DM, Demeester PT, et al. Definition of histopathologic changes in gastroesophageal reflux disease. Am J Surg Pathol 2000;24:344-51.

2. Chandrasoma PT, Der R, Ma Y, et al. Histology of the gastroesophageal junction: an autopsy study. Am J Surg Pathol 2000;24: 402-9.

3. Chen YY, Antonioli DA, Spechler SJ, et al. Gastroesophageal reflux disease versus Helicobacter pylori infection as the cause of gastric carditis. Mod Pathol 1998;11:950-6.

4. Der R, Tsao-Wei DD, Demeester T, et al. Carditis: a manifestation of gastroesophageal reflux disease. Am J Surg Pathol 2001;25: $245-52$.

5. Genta RM, Huberman RM, Graham DY. The gastric cardia in Helicobacter pylori infection. Hum Pathol 1994;25:915-9.

6. Glickman JN, Chen YY, Wang HH, et al. Phenotypic characteristics of a distinctive multilayered epithelium suggests that it is a precursor in the development of Barrett's esophagus. Am J Surg Pathol 2001;25:569-78.

7. Glickman JN, Fox V, Wang HH, et al. Morphology of the cardia and significance of carditis in pediatric patients. Am J Surg Pathol 2002;26:1032-9.

8. Goldblum JR, Vicari JJ, Falk GW, et al. Inflammation and intestinal metaplasia of the gastric cardia: role of gastroesophageal reflux and $H$. pylori infection. Gastroenterology 1998;114: 633-9.

9. Kilgore SP, Ormsby AH, Gramlich TL, et al. The gastric cardia: fact or fiction? Am J Gastroenterol 2000;95:921-4.

10. Law S, Lam KY, Chu KM, et al. Specialized intestinal metaplasia and carditis at the gastroesophageal junction in Chinese patients undergoing endoscopy. Am J Gastroenterol 2002;97:1924-9.

11. Maynten T, Farkkila M, Kunnamo I, et al. The impact of upper GI endoscopy referral volume on the diagnosis of gastroesophageal reflux disease and its complications: a 1-year cross-sectional study in a referral area with 260,000 inhabitants. Am J Gastroenterol 2002;97:2524-9.

12. Nebel OT, Fornes MF, Castell DO. Symptomatic gastroesophageal reflux: incidence and precipitating factors. Am J Dig Dis 1976; 21:953.

13. Shields H, Rosenberg SJ, Zwas FR, et al. Prospective evaluation of multilayered epithelium in Barrett's esophagus. Am J Gastroenterol 2001;96:3268-73.

14. Voutilainen M, Farkkila M, Mecklin JP, et al. Chronic inflammation at the gastroesophageal junction (carditis) appears to be a specific finding related to Helicobacter pylori infection and gastroesophageal reflux disease: the Central Finland Endoscopy Study Group. Am J Gastroenterol 1999;94:3175-80.

15. Wieczorek TJ, Wang HH, Glickman J, et al. Clinical-pathologic distinction between reflux carditis and $\mathrm{H}$. plyori carditis [Abstract]. Mod Pathol 2002;15:148.

16. Wieczorek TJ, Wang HH, Antonioli D, et al. Multilayered epithe- lium is associated with reflux-induced carditis in patients without Barrett's esophagus. Gastroenterology 2002;122:A288.

\section{Histopathology of the Gastroesophageal Junction: A Study on 36 Operation Specimens}

To the Editor:

I read with great interest the recent paper reporting the histologic findings at the gastroesophageal junction in a population of patients with squamous carcinoma of the proximal esophagus. ${ }^{3}$ This population is valuable because it represents a group of patients without significant risk of reflux in whom esophagogastrectomy specimens are available for pathologic examination.

I would like to commend the authors on the absolute precision of their definitions in the "Methods" section of their article. Their method of specimen processing with separation of the specimen at the grossly determined gastroesophageal junction and vertical sectioning of the entire circumference of the junctional region in the proximal gastric pouch is perfect. Their definitions of cardiac mucosa and oxynto-cardiac mucosa and the way they measure these are precise and easily reproducible. It is only when studies adhere to such strictly precise definitions and methods that comparison of data become scientifically feasible. Much of the confusion in the literature that exists today has resulted from a complete lack of standardization of method and definition. Our methods and definitions are identical to those described in this article and I suggest that they become the standard.

The data presented lend considerable support to our concept that cardiac mucosa and oxynto-cardiac mucosa are abnormal, reflux-associated glandular epithelia rather than normal structures. ${ }^{1}$ Even in this older population with esophageal disease (not reflux-associated), 1 of 36 patients had no cardiac mucosa, 15 of 36 patients had cardiac mucosa only in a part of the circumference of the squamocolumnar junction, the median minimal and maximal lengths of cardiac mucosa were $1 \mathrm{~mm}$ and 5 $\mathrm{mm}$, respectively, and the minimal length of cardiac mucosa had a correlation with reflux disease. Even with these short lengths of cardiac and oxynto-cardiac mucosa, the authors produced proof that they were located in the esophagus by the finding of submucosal mucous glands or esophageal gland ducts under these epithelia in 9 of 36 cases.

The authors have interpreted their data with commendable reserve. Their study, however, provides further evidence of shrinkage of what we can accept as the "normal" extent of cardiac mucosa at the gastroesophageal junction. We have suggested that this is zero. Over the past years, the accepted length for cardiac mucosa has decreased from $2+\mathrm{cm}$ to $0.18 \mathrm{~cm}$ in Kilgore et al.'s 
autopsy paper ${ }^{2}$ to "only sometimes present" in this article. ${ }^{3}$ The literature is inexorably heading to the truth of zero for the normal length of cardiac mucosa.

Para Chandrasoma, M.D.

Department of Surgical Pathology

LAC+USC Medical Center

Los Angeles, California, U.S.A.

\section{REFERENCES}

1. Chandrasoma PT, Der R, Ma Y, et al. Histology of the gastroesophageal junction: an autopsy study. Am J Surg Pathol 2000;24: 402-9.

2. Kilgore SP, Ormsby AH, Gramlich TL, et al. The gastric cardia: fact or fiction? Am J Gastroenterol 2000;95:921-4.

3. Sarbia M, Donner A, Gabbert HE. Histopathology of the gastroesophageal junction: a study on 36 operation specimens. Am J Surg Pathol 2002;26:1207-12. 